\begin{tabular}{|c|l|}
\hline Title & A residue formula for the index of a holomorphic flow \\
\hline Author(s) & Seade, J.; Suwa, T. \\
\hline Citation & Hokkaido University Preprint Series in Mathematics, 192, 1-22 \\
\hline Issue Date & 1993-03 \\
\hline DOI & 10.14943/83336 \\
\hline Doc URL & http://hdl.handle.net/2115/68938 \\
\hline Type & bulletin (article) \\
\hline File Information & pre192.pdf \\
\hline
\end{tabular}

Instructions for use 
A RESIDUE FORMULA FOR

THE INDEX OF

A HOLOMORPHIC FLOW

J. Seade and T. Suwa

Series $\sharp 192$. March 1993 


\section{HOKKAIDO UNIVERSITY \\ PREPRINT SERIES IN MATHEMATICS}

\#165: J. Wierzbicki, Y. Watatani, Commuting squares and relative entropy for two subfactors, 18 pages. 1992.

$\sharp 166$ : Y. Okabe, A new algorithm driven from the view-point of the fluctuation-dissipation theorem in the theory of $\mathrm{KM}_{2} \mathrm{O}$-Langevin equations, 13 pages. 1992.

\#167: Y. Okabe, H. Mano and Y. Itoh, Random collision model for interacting populations of two species and its strong law of large numbers, 14 pages. 1992.

$\#$ 168: A. Inoue, On the equations of stationary precesses with divergent diffusion coefficients, 25 pages. 1992.

\# 169: T. Ozawa, Remarks on quadratic nonlinear Schrödinger equations, 19 pages. 1992.

$\sharp 170$ : T. Fukui, Y. Giga, Motion of a graph by nonsmooth weighted curvature, 11 pages. 1992.

$\sharp 171$ : J. Inoue, T. Nakazi, Finite dimensional solution sets of extremal problems in $\mathrm{H}^{1}, 10$ pages. 1992.

$\sharp 172$ : S. Izumiya, A characterization of complete integrability for partial differential equations of first order, 6 pages. 1992.

\#173: T. Suwa, Unfoldings of codimension one complex analytic foliation singularities, 49 pages. 1992.

$\sharp 174$ : T. Ozawa, Wave propagation in even dimensional spaces, 15 pages. 1992.

$\sharp 175$ : S. Izumiya, Systems of Clairaut type, 7 pages. 1992.

$\sharp 176$ : A. Hoshiga, The initial value problems for quasi-linear wave equations in two space dimensions with small data, 25 pages. 1992.

\#177: K. Sugano, On bicommutators of modules over H-separable extension rings III, 9 pages. 1993.

$\sharp 178$ : T. Nakazi, Toeplitz operators and weighted norm inequalities, 17 pages. 1993.

$\sharp 179$ : O. Ogurisu, Existence and structure of infinitely degenerate zero-energy ground states of a Wess-Zumino type model in supersymmetric quantum mechanics, 26 pages. 1993.

$\sharp 180$ : O. Ogurisu, Ground state of a spin $1 / 2$ charged particle in an even dimensional magnetic field, 9 pages. 1993.

$\sharp 181$ : K. Sugano, Note on H-separable Galois extension, 6 pages. 1993.

$\sharp$ 182: M. Yamada, Distance formulas of asymptotic Toeplitz and Hankel operators, 13 pages. 1993

$\sharp 183$ : G. Ishikawa, T. Ozawa, The genus of a connected compact real algebraic surface in the affine three space, 11 pages. 1993.

\#184: T. Hibi, Canonical modules and Cohen-Macaulay types of partially ordered sets, 6 pages. 1993.

$\sharp 185$ : Y. Giga, K. Yama-uchi, On a lower bound for the extinction time of surfaces moved by mean curvature, 16 pages. 1993.

$\sharp 186$ : Y. Kurokawa, On functional moduli for first order ordinary differential equations, 9 pages. 1993.

$\sharp 187$ : O. Ogurisu, Unitary equivalence between a spin 1/2 charged particle in a two-dimensional magnetic field and a spin $1 / 2$ neutral particle with an anomalous magnetic moment in a two-dimensional electric field, 4 pages. 1993.

$\sharp 188$ : A. Jensen, T. Ozawa, Existence and non-existence results for wave operators for perturbations of the laplacian, 30 pages. 1993 .

$\sharp 189$ : T. Nakazi, Multipliers of invariant subspaces in the bidisc, 12 pages. 1993.

$\sharp 190$ : S. Izumiya, G.T. Kossioris, Semi-local classification of geometric singularities for Hamilton-Jacobi equations, 24 pages. 1993.

$\sharp 191$ : N. Hayashi, T. Ozawa, Finite energy solutions of nonlinear Schrödinger equations of derivative type, 21 pages. 1993. 


\title{
A RESIDUE FORMULA FOR THE INDEX OF A HOLOMORPHIC FLOW\#
}

\author{
JOSE SEADE and TATSUO SUWA
}

Let $(V, P)$ be a normal, isolated complex analytic singularity of dimension $n \geq 1$, and consider germs of holomorphic vector fields on $V$ at $P$. One would like to be able to distinguish different germs.

If $P$ is a regular point of $V$, there are a number of invariants that sometimes allow us to distinguish germs of vector fields at $P$; However, for $P$ singular, little is known.

If $X$ is a holomorphic vector field on $V$, singular only at $P$, then a natural way for studying the geometry, the topology and the dynamics of $X$ near $P$, is to go up to a resolution of $P$ and see what happens there, see for instance [HH]. More precisely, let

$$
\pi: \tilde{V} \rightarrow v,
$$

be a resolution of $P$ and let $E=\pi^{-1}(P)$ be the exceptional set of $\pi$. The solutions of $X$ determine a non-singular holomorphic foliation $\mathcal{F}$ on $V-\{P\}$ by immersed Riemann surfaces. The strict transform of $\mathcal{F}$ is a holomorphic foliation $\tilde{F}$ of $\tilde{V}$ by complex curves, with singular set $S$ contained in $E$. Since $\pi$ is a biholomorphism on $\tilde{V}-E$, the vector field $X$ lifts to a vector field $\tilde{X}$ on $\tilde{V}-E$, tangent to $\tilde{F}$. Thus one has several types of invariants to look at:

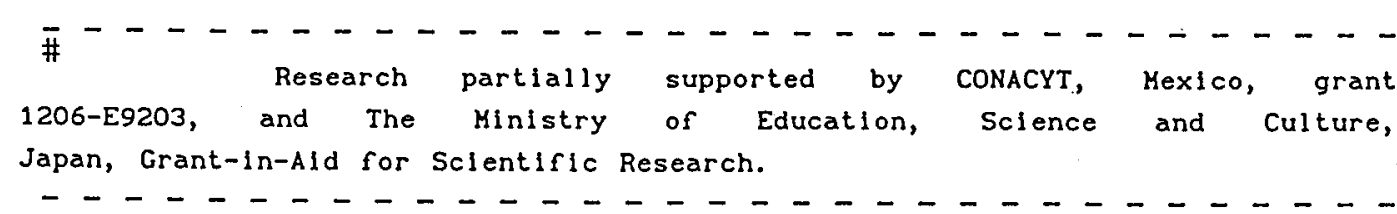


a) We may forget that $\tilde{X}$ is holomorphic and think of it as a $C^{\infty}$-vector field. As such, we can approximate it by a sequence of vector fields $\left\{Y_{t}\right\}$, defined on all of $\tilde{v}$ and having non-degenerate singularities. The total number of singular points of the $Y_{t}^{\prime} s$, counted with "signs", is independent of the way we approximate $\tilde{\mathrm{X}}$ : This is the (tatal) index Ind $(\tilde{\mathrm{X}}, \tilde{V})$ of $\tilde{\mathrm{X}}$ in $\tilde{V}$, introduced by Poincaré-Hopf, see [Mi].

b) The bundle $\mathrm{T} \tilde{F}$, tangent to $\tilde{F}$ on $\tilde{V}-S$, can be extended to a holomorphic line bundle $L$ over all of $\tilde{v}$. Thus one has the Chern class $c_{1}(L)=c_{1}(T \tilde{F}) \in H^{2}(\tilde{V} ; \mathbb{Z})$. This is a topological invariant of $\tilde{F}$, by [GSV]. One also has the narmal sheaf $Q$ of $\tilde{F}$, defined by $Q=T \tilde{V}_{L}$. The Chern classes of $Q$, $c_{i}(Q) \in H^{2 i}(\tilde{V} ; \mathbb{Z}), \quad i=1, \ldots, n$, are linear combinations of products of the Chern classes of $T \tilde{V}$ and the class $c_{1}(L)$, see [AH] or $\S 2$ below.

c) One may consider the top Chern class $c_{n}(Q)$ of the normal sheaf. He know, see $\$ 2$ below, that the Chern class $c_{n}(Q)$ can be considered as a relative class in $H^{2 n}(\tilde{V}, \tilde{V}-E ; \mathbb{Z})$. Essentially by [BB], this class is localized at the singular set $S \subset E$ :

$$
c_{n}(Q)=\sum_{Z \subset S} \mu_{*} \operatorname{Res} c_{n}(\tilde{F}, Z),
$$

where the sum runs over the connected components of $S, \operatorname{Res}_{c_{n}}(\tilde{F}, Z)$ is a homology class in $\mathrm{H}_{0}(Z ; \mathbb{Z})$ that depends only on $\tilde{F}$ near $Z$, and $\mu_{*}$ is given by the inclusion $\mathrm{H}_{0}(Z ; \mathbb{Z})$ into $\mathrm{H}_{0}(\tilde{V} ; \mathbb{Z})$, followed by Poincaré-Lefschetz duality $\mathrm{H}_{0}(\tilde{V} ; \mathbb{Z}) \cong \mathrm{H}^{2 \mathrm{n}}(\tilde{V}, \tilde{V}-E ; \mathbb{Z})$. If $P_{0}$ is an isolated singularity of $\tilde{F}$, the term $\operatorname{Res}_{C_{n}}\left(\tilde{F}, P_{0}\right)$ is the corresponding Grothendieck residue, by $[B B]$. Thus it equals the local Milnor number

$$
\mu=\frac{\operatorname{dim} o_{n, P_{0}}}{\left(F_{1}, \ldots, F_{n}\right)},
$$

where the $F_{i}^{\prime} s$ are holomorphic functions that define the germ of $\tilde{F}$ at $P_{0}$, c.f. [CLS, CCS]. 
Several questions arise:

Q. 1. Is it possible to evaluate the index $\operatorname{Ind}(\tilde{\mathrm{X}}, \tilde{V})$ in terms of the above invariants of $\tilde{F}$ ?.

Q. 2. Can we use all these invariants to define something which depends only on the germ $(V, P)$ and the vector field $X$, and not on the choice of resolution of $P$ ?.

Q. 3 If so, what kind of invariant is this?.

In this work we study and partially answer these questions. Question 1 is a special case of a more general question: Let $W$ be a connected, complex manifold, which is the interior of a compact, $C^{\infty}$-manifold with boundary $M=\partial W$. Let $X$ be a holomorphic vector field, defined and non-singular, on a neighbourhood $U$ of $M$ in $W$. Assume further that $X$ is tangent on $u$, to a 1-dimensional holomorphic foliation $F$ defined on $W$ and with compact singular locus $S \subset W-U$. Are the invariants, or "residues", of Baum-Bott [BB] related to the Poincaré-Hopf index of $X$ ?. Theorem I below gives an answer to this question, and also to question 1 above (see $\S 2$ ).

THEOREM I : Let $X$ be a holomorphic vector field, defined and non-singular on a neighbourhood $u$ of $\partial w$, the boundary of a complex manifold $W$ which is the interior of a compact, smooth manifold with non-empty boundary. Suppose the holomorphic foliation defined by $x$ on $u$ can be extended to a holomorphic foiliation $F$ on $W$, which is full in the sense of [BB]. (So its singular set $S$ has codimension at least 2.) Then the total (Poincaré-Hopf) index of $X$ in $W$ is :

$$
\operatorname{Ind}(\mathrm{X}, \mathrm{W})=\tilde{c}_{1}(F) \cdot \mathrm{c}_{\mathrm{n}-1}(Q)[W]+\sum_{\mathrm{ZCS}} \mu_{*} \operatorname{Res}_{\mathrm{c}_{\mathrm{n}}}(\mathcal{F}, Z)[W],
$$

where $\tilde{c}_{1}(\mathscr{F})$ is the Chern class of $\mathscr{F}$ relative to $X .,[W]$ is the fundamental cycle and the sum on the right runs over the connected components of the singular set $S$. In particular, if 
the singular set $S$ consists of isolated points $P_{1}, \ldots, P_{r}$, then

$$
\operatorname{Ind}(\mathrm{X}, W)=\tilde{c}_{1}(\mathcal{F}) \cdot \mathrm{c}_{n-1}(Q)[W]+\sum_{i=1}^{r} \mu_{i},
$$

where $\mu_{i}$ is the local Milnor number of $\mathscr{F}$ at the singular point $P_{i}, i=1, \ldots, r$.

Theorem II is of a different nature, closer to singularities theory. In fact Theorem II is a consequence of a deep theorem of Laufer, Steenbrink and Looijenga, see $\$ 1$ below. This gives an answer to questions 2 and 3 above, for complete intersection germs of even dimension. The term $\mathrm{Td}^{*}$ is a ratinal linear combination of the Chern numbers of $\tilde{v}$.

THEOREM II. Let $\mathrm{n}=2 \mathrm{k}$ be even and let $\mathrm{q}_{\mathrm{n}}=\frac{(-1)^{\mathrm{k}-1} \mathscr{B}_{\mathrm{k}}}{2 \mathrm{k} \text { ! }}$, where $\mathcal{B}_{k}$ is the $k^{\text {th }}$-Bernoulli number, see $[\mathrm{Hr}]$. Let $(V, P)$ be an isolated complete intersection singularity (ICIS) of dimension $\mathrm{n}$, and let $\mathrm{x}$ be a continuous vector field on $v$, non-singular away from $P$. If $\tilde{X}$ is the lifting of $X$ to a resolution $\tilde{V}$ of $P$, then the rational number

$$
\text { Ind }(\tilde{X}, \tilde{V})+\frac{1}{q_{n}} \operatorname{Td}^{*}[\tilde{V}],
$$

depends only on the germ of $V$ at $P$ and the homotopy class of the vector field $X$ near $P$, independently of the choice of resolution of $\mathrm{P}$; $\operatorname{Td}^{*}[\tilde{V}]$ is (see $\S 1$ below) the $\mathrm{n}^{\text {th }}$ Todd polynomial $T d_{n}$ in the relative Chern numbers of $\tilde{V}$, but taking $c_{n}=0$. Furthermore,

$$
\operatorname{Ind}(\mathrm{X}, V)=\operatorname{Ind}(\tilde{X}, \tilde{V})+\frac{1}{q_{\mathrm{n}}}\left(\mathrm{Td}^{*}[\tilde{V}]+\mathrm{p}_{g}\right),
$$

where Ind $(X, V)$ is the index of $X$ on $V$ at $P$ and $p_{g}$ is the geometric genus of $P$. 
If $P$ is regular in $V$, Ind $(X, V)$ is the usual local index of Poincaré-Hopf; In general, if $P$ is an ICIS, this is essentially the local index of a vector field on a singular variety, studied in [GSV, BG], see $\$ 1$ below. The number $q_{n}$ above is the coefficient of $c_{n}$ in the $n^{\text {th }}$ - Todd polynomial $\mathrm{Td}_{n}$, by [BH]. For $\mathrm{n}>1$ odd, the coefficient of $c_{n}$ in $\mathrm{Td}_{n}$ is 0 and this is why our method says nothing in these dimensions. For an ICIS of dimension 1 in $\mathbb{C}^{k}$, the statement corresponding to Theorem II is:

$$
\operatorname{Ind}(\mathrm{X}, V)=\operatorname{Ind}((\tilde{\mathrm{X}}, \tilde{V})-2 \delta \text {, }
$$

where $\delta>0$ "measures" the number of double points concentrated at $P$, see [Mi p.85, or Se p.68], and Ind $(X, V)$ is the index of $X$ on $V$, so it is the element in $\pi_{1}(U(k)) \cong \mathbb{Z}$ determined by the map $(V-\{P\}) \rightarrow U(k)$ given by $X$ and the gradient vector fields of the functions that define the germ of $V$ at $P$, c.f. $\$ 1$ below. (This follows from Milnor's formula,

$$
\mu-1=2 \delta-r \text {. }
$$

where $\mu$ is the Milnor number and $r$ is the number of irreducible components of $V$ at $P$, and the proof is the same as that of Theorem II, replacing the theorem of Laufer, Steenbrink and Looijenga by the above formula of Milnor.)

Theorem III is the sum of Theorems I and II :

THEOREM III. Let $n=2 k$ be even. Let $q_{n}$ be as in theorem II. Let $(V, P)$ be an ICIS of dimension $n$, and let $X$ be a holomorphic vector field on $V$, non-singular away from $P$. If $\tilde{F}$ is the strict transform of the foliation on $V-\{P\}$ determined by $X$, then the index of $X$ on $V$ at $P$ is: $\operatorname{Ind}(X, V)=\sum_{Z C S} \mu_{*} \operatorname{Res}{ }_{c_{n}}(\tilde{F}, Z)[\tilde{V}]+\tilde{c}_{1}(\tilde{F}) \cdot c_{n-1}(Q)[\tilde{V}]+\frac{1}{q_{n}}\left(T d^{*}[\tilde{V}]+p_{g}\right)$. In particular, if the singularities of $\tilde{F}$ are isolated, then the first term on the right hand side is the sum of the local Milnor numbers. 
For instance, if $\mathrm{n}=2$, the singularities of $\tilde{F}$ are necessarily isolated, the coefficient $q_{n}$ is $\frac{1}{12}$ and $\operatorname{Td}^{*}[\tilde{V}]$ is $\frac{K^{2}}{12}$, where $x$ is the canonical class of the resolution. Thus the formula above becomes,

$$
\operatorname{Ind}(\mathrm{X}, v)=\tilde{c}_{1}(F) \cdot c_{1}(Q)[W]+\sum_{1=1}^{r} \mu_{i}+\mathrm{K}^{2}+12 \cdot \mathrm{p}_{g} .
$$

In a subsequent work [SS], we, study some natural generalizations of $\$ 2$ above: What happens when the dimension of the leaves of $F$ is larger than 1 ?. What if we have, on a neighbourhood of the boundary, several vector fields tangent to F ? , can we say anything for characteristic classes of $W, F, Q$, other than $c_{n}$ ?. Lemma 2.1 below contains a result in this direction.

This work started when both authors were participating in the "College on singularities" at the ICTP, Trieste, and it was essentially finished when the first named author visited the Hokkaido University . The first named author also visited IMPA, at Río de Janeiro, while working on it. The authors want to thank these institutions for their support and hospitality. 


\section{§1. VECTOR FIELDS AND THE TODD GENUS.}

We recall [Hr] that the Todd sequence $\left\{\mathrm{Td}_{\mathrm{k}}\right\}, \mathrm{k} \in \mathbb{N}$, is the (multiplicative) sequence of polynomials associated to the power series $\frac{x}{1-e^{-x}}$. This is a sequence of polynomials in indeterminates $c_{1}, \ldots, c_{k}, \ldots$, such that $T_{k}$ is a rational linear combination of $c_{1}, \ldots, c_{k}$, and if we assign the weight i to $c_{i}$ and weight $i_{1}+\ldots+i_{r}$ to the cup product $c_{i_{1}} \cdot \ldots \cdot c_{i_{r}}$, then $\operatorname{Td}_{k}$ is homogeneous of degree $k$. For example,

$T d_{1}=\frac{1}{2} \quad c_{1}$

$\mathrm{Td}_{2}=\frac{1}{12}\left(\mathrm{c}_{1}^{2}+\mathrm{c}_{2}\right)$,

$\mathrm{Td}_{3}=\frac{1}{24} \mathrm{c}_{1} \mathrm{c}_{2}$,

$\mathrm{Td}_{4}=\frac{1}{720}\left(-c_{4}+c_{3} c_{1}+3 c_{2}^{2}+4 c_{2} c_{1}^{2}-c_{1}^{4}\right)$,

and so on. Let us denote by $\mathrm{Td}_{n}^{*}$ the polynomial obtained from the $n^{\text {th }}$-Todd polynomial $T_{n}$, replacing $c_{n}$ by 0 . In other words, we write $T d_{n}=T d_{n}^{*}+q_{n} c_{n}$, where $q_{n}$ is the coefficient of $c_{n}$ in $\mathrm{Td}_{n}$. Thus, for instance, $\operatorname{Td}_{2}^{*}=\frac{1}{12} \cdot c_{1}^{2}$. (We refer to [Hr or M3] for basic material about the Todd genus.)

If $W$ is a closed, connected, $C^{\infty}$-manifold of (real) even dimension $2 \mathrm{n}$, and a complex structure on its tangent bundle $\mathrm{TW}$, its Todd genus is defined by,

$$
\operatorname{Td}[W]=\operatorname{Td}_{n}\left(c_{1}, \ldots, c_{n}\right)[W] \text {, }
$$

where $c_{1}, \ldots, c_{n}$ are the Chern classes of $T W$ and $[W]$ is the fundamental cycle. If $W$ has non-empty boundary $M$, then $H^{2 n}(W) \cong 0$, so it is not interesting to speak of the Chern numbers of $W$. However, let us suppose we are given a $C^{\infty}$-vector field $X$, defined and non-singular on a neighbourhood of the boundary M. We may look at the primary obstruction for extending this to a cross section of TW. This is a relative cohomology class 
$\tilde{c}_{n}(X) \in H^{2 n}(W, M ; \mathbb{Z})$ that maps to the usual Chern class of TW. and evaluated on the fundamental cycle [W] equals the total index of $X$ in $W$. There are as many liftings of $c_{n}$ to a relative class as elements in $\mathrm{H}^{2 n-1}(M) \cong \mathbb{Z}$, parametrizing the possible indices that the vector fields as above can have. Moreover, if the bundle $\left.T W\right|_{M}$ is trivial, then the Chern classes of TW vanish over the boundary $M$, hence each Chern class $c_{i}(W)$ can be lifted to a relative class $\tilde{c}_{i}(W)$. In general, there will be many different liftings of each $c_{i}(W)$; However, as noted in [LS] or [L1], if $i, j$ are both $\geq 1$ then the cup product $\tilde{c}_{i}(W) \cdot \tilde{c}_{j}(W)$ depends only on the absolute classes $c_{i}(W), c_{j}(W)$. Therefore, the Chern numbers of $W$ involving the lower dimensional classes $c_{1}, \ldots, c_{n-1}$, are well defined, depending only on $W$. In this situation one may define the godd genus of $W$ relative to $X$ by

$$
\operatorname{Td}[W ; X]=\operatorname{Td}\left(\tilde{c}_{1}, \ldots, \tilde{c}_{n-1}, \tilde{c}_{n}(X)\right)[W],
$$

where $T d n$ is the $n^{\text {th }}$-Todd polynomial, $\tilde{c}_{n}(X)$ is the relative Chern class determined by $X$ and the term on the right means that whenever we have a monomial of $\mathrm{Td}_{n}$ which is a product $c_{i_{1}} \cdot \ldots \cdot c_{i_{r}}$, we replace this by the lift $\tilde{c}_{i_{1}} \cdot \ldots \cdot \tilde{c}_{i_{r}}$.

Let us now denote by $(V, P)$ an isolated, complete intersection singularity $(V, P)$ of complex dimension $n \geq 1$. For simplicity, we call such a germ an ICIS, following [L2]. Let $\tilde{V}$ be a resolution of $(V, P)$; This will play the role of the above manifold $W$. By restricting the projection $\pi: \tilde{V} \rightarrow V$ to a compact neighbourhood of $P$, we may think of $\tilde{V}$ as being a compact 2 -manifold with boundary $M$, the link of $P$ in $V$, and with a complex structure on its interior. Let $c_{i} \in H^{2 i}(\tilde{V} ; \mathbb{Z}), i=1, \ldots, n$, be the Chern classes of $\tilde{V}$. A vector field $X$ on $(V, P)$ determines a vector field $\tilde{X}$ on a neighbourhood of the boundary of $\tilde{v}$; The above discussion tells us that $\tilde{X}$ determines a lift $\tilde{c}_{n}(\tilde{X})$ of $c_{n}$ to a relative class, 
with $\tilde{c}_{n}(\tilde{X})[\tilde{V}]=\operatorname{Ind}(\tilde{X}, \tilde{V})$. The tangent bundle $T(V-\{P\})$ is a (smoothly) trivial bundle, because $P$ is an ICIS, hence $\left.T \tilde{V}\right|_{M}$ is smoothly trivial. Thus one has the Jodd genus of $\tilde{V}$ relative to $\tilde{\mathrm{X}}$ :

$$
\operatorname{Td}[\tilde{V} ; \tilde{X}]=\operatorname{Td}\left(\tilde{c}_{1}, \ldots, \tilde{c}_{n-1}, \tilde{c}_{n}(\tilde{X})\right)[\tilde{V}] \text {, }
$$

where $T d_{n}$ is the $n^{\text {th }}$-Todd polynomial, $\tilde{c}_{n}(\tilde{X})$ is the relative Chern class determined by $\tilde{X}$ and the term on the right means that whenever we have a monomial of $T d_{n}$ which is a decomposable product $c_{i_{1}} \cdot \cdots \cdot c_{i_{r}}$, we replace this by the lift $\tilde{c}_{i_{1}} \cdot \ldots \cdot \tilde{c}_{i_{r}}$.

We remark that $\operatorname{Td}[\tilde{V}, \tilde{X}]$ can be expressed as,

$$
\operatorname{Td}[\tilde{V}, \tilde{X}]=q_{n} \cdot \operatorname{Ind}(\tilde{X}, \tilde{V})+\operatorname{Td}^{*}[\tilde{V}],
$$

where $\operatorname{Td}^{*}[\tilde{V}]=\operatorname{Td}_{n}^{*}\left(\tilde{c}_{1}, \ldots, \tilde{c}_{n-1}, 0\right)[\tilde{V}]$ depends only on $\tilde{V}$, not on $X$, and $q_{n}$ is the coefficient of $c_{n}$ in the Todd polynomial $\mathrm{Td}_{\mathrm{n}}$. If $\mathrm{n}>1$ is odd, then $\mathrm{q}_{\mathrm{n}}$ is zero, by [Hr]. For $\mathrm{n}=1, \mathrm{q}_{\mathrm{n}}$ is $\frac{1}{2}$ and for $\mathrm{n}=2 \mathrm{k}, \mathrm{q}_{\mathrm{n}}$ is $\frac{(-1)^{\mathrm{k}-1} \mathcal{B}_{\mathrm{k}}}{2 \mathrm{k} !}$, where $B_{k}$ is the $k^{\text {th }}$-Bernoulli number, see $[\mathrm{Hr}, \mathrm{BH}$.

If $f_{1}, \ldots, f_{r}$ are holomorphic functions that define the germ $(V, P)$, then the gradient vector fields $\nabla f_{i}$ of the $f_{i}^{\prime} s$ are linearly independent over $\mathbb{C}$ away from $P$, because $P$ is an ICIS, and they are orthogonal to $v$. Hence, up to homotopy these vector fields define a smooth map,

$$
\left.\left(X, \nabla f_{1}, \ldots, \nabla f_{r}\right)\right|_{M}: M \rightarrow W_{r+1, r+n},
$$

where $W_{r+1, r+n}$ is the Stiefel manifold of complex orthonormal $(r+1)$-frames in $\mathbb{C}^{n+r} ; W_{r+1, r+n}$ is $(2 n-2)$-connected and its first non-zero homotopy group is $\pi_{2 n-1}\left(W_{r+1, r+n}\right) \cong \mathbb{Z}$, by Bott's computations of the homotopy groups of the classical groups, see for instance [Hu]. Hence the maps from $M$ into $W_{r+1, r+n}$ have a degree. Following [GSV], define the lacal index of $X$ an $v$ at $P$ to be the degree of the above map $\left(X, \nabla f_{1}, \ldots, \nabla f_{r}\right)$. This definition of the local index on a singular variety was introduced 
in [GSV] for hypersurfaces, but the definition and its basic properties are essentially the same (c.f. [BG]).

THEOREM II. Let $n=2 k$ be even and let $q_{n}$ be the coefficient of $c_{n}$ in $T d_{n}$. Let $(V, P)$ be an ICIS of dimension $n$, and let $X$ be a vector field on $V$, non-singular away from $P$. If $\tilde{X}$ is the lifting of $X$ to a resolution $\tilde{V}$ of $P$. Then the rational number

$$
\operatorname{Ind}(\tilde{X}, \tilde{V})+\frac{1}{q_{n}} \operatorname{Td}^{*}[\tilde{V}],
$$

depends only on the germ of $V$ at $P$ and the homotopy class of the vector field $X$, independently of the choice of resolution of $P$. Furthermore,

$$
\operatorname{Ind}(\mathrm{X}, v)=\operatorname{Ind}(\tilde{X}, \tilde{V})+\frac{1}{q_{\mathrm{n}}}\left(\operatorname{Td}^{*}[\tilde{V}]+\mathrm{p}_{\mathrm{g}}\right),
$$

where Ind $(X, V)$ is the index of $X$ at $P$ and $p_{g}$ is the geometric genus of $P$.

This theorem follows from the Laufer-Steenbrink-Looijenga theorem (see [L1]), that we re-formulate below without proof, together with lemmas 1.2 and 1.3 below. The theorem in [L1] is in fact more general than we state below, but this is what we need.

THEOREM [L1]. Let $(V, P)$ be an ICIS of dimension $n$, let $F$ be a Milnor fibre of $P$ and let $\tilde{V}$ be a resolution of $P$. Let $\chi(F)$ and $\chi(\tilde{V})$ be the topological Euler-Poincaré characteristics of $F$ and $\tilde{v}$, respectively. Then,

where $\operatorname{Td}^{*}[\tilde{V}]$ and $\mathrm{p}_{\mathrm{g}}$ are as above.

$$
q_{n} \cdot \chi(F)=q_{n} \cdot \chi(\tilde{V})+T d^{*}[\tilde{v}]+(-1)^{n} p_{g} \text {. }
$$


1.2 LEMMA. Let $X$ be a vector field on $V$, which is everywhere transversal to the link $M$ of $P$ in $V$. Then, $\operatorname{Ind}\left(X^{\prime}, v\right)=\chi(F)$.

PRoOF. The link $M$ of $P$ in $V$ is, by definition, the intersection of $V$ with a small sphere $S_{\varepsilon}$ around $P$. From [M1], or using Ehresman fibration theorem, one knows that $M$ is isotopic in $S_{\varepsilon}$ to the intersection of $S_{\varepsilon}$ with a non-singular fibre $\quad F^{\prime}=f_{1}^{-1}(t) \cap . . n f_{r}^{-1}(t)$, for suitable $t$. Thus, the vector field $X^{\prime}$ can be regarded as a continuous vector field on a neighbourhood of $F^{\prime} \cap S_{\varepsilon}$ in $F^{\prime}$. Let $F$ be $F^{\prime} \cap D_{\varepsilon}$, a Milnor fibre of $P$. Then the gradient vector fields $\nabla f_{1}, \ldots, \nabla f_{r}$ are linearly independent everywhere on $F$, because $(V, P)$ is an ICIS. Hence, the obstruction for extending the map,

$$
\left.\left(X^{\prime}, \nabla f_{1}, \ldots, \nabla f_{r}\right)\right|_{M}: M \rightarrow W_{r+1, r+n},
$$

to all of $F$ is the obstruction for extending $X^{\prime}$ to a vector field on $F$. Since the degree of this map is the index of $X^{\prime}$ on $V$ at $P$, it follows that Ind $(X, V)$ can be regarded as the obstruction for extending $X^{\prime}$ to a continuous vector field on $F$. The lemma now follows from the theorem of Poincaré-Hopf for manifolds with boundary, because $X^{\prime}$ is everywhere transversal to the boundary of $F$.

The following lemma is an immediate consequence of lemma 1.2 and a well known result in differential topology, saying that the difference between the indices of two vector fields on a manifold with boundary is independent of the topology of the manifold away from a tubular neighbourhood of the boundary. (See for instance [BG].) 
1.3 LEMMA. Let $X$ and $X^{\prime}$ be vector fields on $V$. Then, $\operatorname{Ind}(X, V)-\operatorname{Ind}\left(X^{\prime}, V\right)=\operatorname{Ind}(\tilde{X}, \tilde{V})-\operatorname{Ind}\left(\tilde{X}^{\prime}, \tilde{V}\right)$

where Ind $(\tilde{X}, \tilde{V})$ and Ind $\left(\tilde{X}^{\prime}, \tilde{V}\right)$ are, respectively, the indices of the liftings of $X$ and $X$ ' to the resolution $\tilde{V}$.

Theorem II now follows easily: By the theorem quoted above one has,

$$
q_{n} \cdot \chi(F)=q_{n} \cdot \chi(\tilde{V})+T d^{*}[\tilde{V}]+(-1)^{n} p_{g} .
$$

If $X^{\prime}$ is a vector field as in lemma 1.2 and $\tilde{X}^{\prime}$ is its lifting to $\tilde{V}$, then the theorem of Poincaré-Hopf for manifolds with boundary [M2] implies Ind $\left(\tilde{X}^{\prime}, \tilde{V}\right)=\chi(\tilde{V})$. Hence lemma 1.2 yields,

$$
\mathrm{q}_{\mathrm{n}} \cdot \operatorname{Ind}\left(\mathrm{X}^{\prime}, v\right)=\mathrm{q}_{\mathrm{n}} \cdot \operatorname{Ind}\left(\tilde{\mathrm{X}}^{\prime}, \tilde{v}\right)+\mathrm{Td}^{*}[\tilde{v}]+(-1)^{\mathrm{n}} \mathrm{p}_{\mathrm{g}} \cdot
$$

If $n$ is even, then $q_{n} \neq 0$ and one has,

$$
\text { (1.4) } \operatorname{Ind}\left(\mathrm{X}^{\prime}, V\right)=\operatorname{Ind}\left(\tilde{X}^{\prime}, \tilde{V}\right)+\frac{1}{\mathrm{q}_{\mathrm{n}}}\left(\mathrm{Td}^{*}[\tilde{V}]+\mathrm{p}_{\mathrm{g}}\right) \text {, }
$$

proving Theorem II for a vector field $X$ ' transversal to the link. If $X$ is a vector field on $V$ as in Theorem II, then

(1.5) $\operatorname{Ind}(X, v)-\operatorname{Ind}\left(X^{\prime}, V\right)=\operatorname{Ind}(\tilde{X}, \tilde{V})-\operatorname{Ind}\left(\tilde{X}^{\prime}, \tilde{V}\right)$,

by lemma 1.3. By 1.4 one has,

$$
\operatorname{Ind}\left(\tilde{X}^{\prime}, \tilde{V}\right)=\operatorname{Ind}\left(X^{\prime}, V\right)-\frac{1}{q_{n}}\left(\operatorname{Td}^{*}[\tilde{V}]+p_{g}\right),
$$

which together with 1.5 implies,

$$
\text { (1.6) } \operatorname{Ind}(\mathrm{X}, v)=\operatorname{Ind}(\tilde{\mathrm{X}}, \tilde{V})+\frac{1}{\mathrm{q}_{\mathrm{n}}}\left(\operatorname{Td}^{*}[\tilde{V}]+\mathrm{p}_{\mathrm{g}}\right) \text {, }
$$

as claimed in Theorem II. Finally, that

$$
\operatorname{Ind}(\tilde{X}, \tilde{V})+\frac{1}{q_{n}} \operatorname{Td}^{*}[\tilde{V}] \text {, }
$$

is independent of the choice of resolution follows from 1.6 and the fact that Ind $(\mathrm{X}, v)$ is defined intrisically, together with the well known fact that the geometric genus $\mathrm{p}_{g}$ is an invariant of $P$. 
§2. FOLIATIONS ON MANIFOLDS WITH BOUNDARY.

Let $\vec{W}$ be a compact, connected manifold with boundary $\partial W \neq \varnothing$, and a complex structure on its interior $W$. So $W$ is a complex manifold; We let $n$ be the complex dimension of $W$ and we assume $n>1$. Let $X$ be a nowhere-vanishing holomorphic vector field on a neighbourhood $u$ of $\partial W$. So $X$ defines a non-singular holomorphic foliation $F=F(X)$ on $U$ by complex curves; We further assume that $F$ is extended to a holomorphic foliation on $W$, with singular set $S$ of codimension $\geq 2$. In practice we will take $W$ to be the resolution $\tilde{V}$ of an ICIS $(V, P)$ and $F$ the strict transform of the foliation on $V-\{P\}$ determined by a holomorphic vector field, as in $\$ 1$ above.

Let $T F$ be the bundle tangent to $F$ on $W-S$; so $T F$ is a holomorphic line sub-bundle of $\mathrm{TW} / \mathrm{W}-\mathrm{S}$. This can always be extended to a holomorphic line bundle $L$ over $W$, which is not a sub-bundle of TW on $S$ : there is a bundle morphism $\pi: L \rightarrow T W$, which is not injective on $S$. The isomorphism class of $L$ is determined by $F$; In fact, as a $C^{\infty}$-vector bundle, $L$ is classified by its first Chern class, and this class depends only on the topological type of $\mathscr{F}$, by [GSV]. This is called the Chern class of the foliation and denoted $c_{1}(F)$. We note that the vector field $X$ trivializes the bundle $L \cong T F$ on $a$ neighbourhood $u$ of the boundary $\partial W$. Thus we have a connection $D_{1}^{\prime}$ for $L$ on $U$, flat relative to the frame $X ; D_{1}^{\prime}$ can be extended to a connection $D_{1}$ for $L$ on $W$, flat on $U$. This determines a representative of the Chern class $c_{1}(F)$ that vanishes over $\partial W$, so it represents a class $\tilde{c}_{1}(\mathscr{F}) \in \mathrm{H}^{2}(W, \partial W ; \mathbb{Z})$. (The Chern classes of vector bundles defined via differential forms live in the cohomology with complex coefficients, but they are in fact integral classes.) 
We now think of $L$ and $T W$ as being locally free sheaves on $W$ of ranks 1 and $n$, respectively. The bundle map $\pi$ induces an injection on the sheaf level and we have the normal sheaf $Q$, defined by the exact sequence of sheaves,

$$
\text { (*) } \quad 0 \rightarrow \mathrm{L} \rightarrow \mathrm{TW} \rightarrow Q \rightarrow 0 \text {. }
$$

We note that $Q$ is not locally free at the singular set $S$, but the exact sequence above provides a resolution of $Q$ by vector bundles. Thus $[A H]$, the total Chern class of $Q$ is:

where $c(L)=1+c_{1}(F)$, because $L$ has rank 1 .

$$
\begin{aligned}
& c(Q)=\frac{c(T W)}{c(L)} \text {, because } L \text { has rank } 1 .
\end{aligned}
$$

2.1 LEMM: Let $\phi=\phi\left(c_{1}, \ldots, c_{n}\right)$ be a polynomial in indeterminates $c_{1}, \ldots, c_{n}$, with complex coefficients, homogeneous of degree $n$. Then, there exist relative cohomology classes $\tilde{\phi}(Q)$ and $\tilde{\phi}(T W)$ in $H^{2 n}(W, \partial W ; \mathbb{C})$, such that:

a) $\tilde{\phi}(Q)$ is localized at the singular set $S$ of $\mathscr{F}$ : $\tilde{\phi}(Q)=\sum_{Z \subset S} \mu_{*}\left(\operatorname{Res}_{\phi}(\mathcal{F}, Z)\right)$.

where the sum runs over the connected components of the singular set, $\operatorname{Res}_{\phi}(\mathcal{F}, Z) \in \mathrm{H}_{0}(Z ; \mathbb{C})$ is the Baum-Bott residue corresponding to the polynomial $\phi ; \mu_{*}$ is the composition $\alpha i_{*}$, where $i_{*}: \mathrm{H}_{0}(Z ; \mathbb{C}) \rightarrow \mathrm{H}_{0}(W ; \mathbb{C})$ is induced by the inclusion and $\alpha: H_{0}(W ; \mathbb{C}) \rightarrow H^{2 n}(W, \partial W ; \mathbb{C})$ is Lefschetz duality.

b) One has,

$$
\tilde{\phi}(T W)=\tilde{\phi}(Q)+\phi^{\prime}(Q, F) \cdot \tilde{c}_{1}(F),
$$

where $\phi^{\prime}(Q, \mathcal{F})$ is a class in $\mathrm{H}^{2 \mathrm{n}-2}(W ; \mathbb{C})$ depending on $c_{1}(Q), \ldots, c_{n-1}(Q)$ and $\tilde{c}_{1}(F)$, and it is computable from $\phi$.

c) Let $\tilde{c}_{n}(T W ; X) \in H^{2 n}(W, M ; \mathbb{Z})$ be the relative Chern class determined (in $\S 1$ ) by the vector field $X$. Then,

$$
\tilde{c}_{n}(T W ; X)=\tilde{c}_{n}(Q)+c_{n-1}(Q) \cdot \tilde{c}_{1}(F)
$$


One may also add that the image of $\tilde{\phi}(Q)$ and $\tilde{\phi}(T W)$ in the absolute cohomology are the corresponding classes $\phi(Q)$ and $\phi(T W)$, however $H^{2 n}(W ; \mathbb{C}) \cong 0$, so this observation is not interesting.

PROOF. We first recall from [BB] the construction of a "basic connection". On W-S the foliation $F$ acts on the normal sheaf Q: If

$$
\eta: T W \rightarrow Q \text {, }
$$

is the projection, define on $W-S$,

$$
\begin{array}{clc}
\mathrm{TF} \times Q & \longrightarrow \\
(\mathrm{u}, \eta(v)) & \longrightarrow & Q \\
& \longrightarrow & \longrightarrow
\end{array}
$$

which is well defined because $\mathrm{TF}$ is closed under Lie brackets. Thus one has a "partial connection" on $Q$,

$$
\delta: C^{\infty}(Q) \longrightarrow C^{\infty}\left(\left(T \mathscr{F} \oplus \vec{T}^{*}\right)^{*} \otimes Q\right)=C^{\infty}\left(\operatorname{Hom}(T \mathscr{F}, Q) \oplus\left(\bar{T}^{*} W \otimes Q\right)\right),
$$

defined by $\delta(s)=(u \rightarrow[u, s], \bar{\partial} s)$, where $\bar{T} W$ is the conjugate of TW in its complexification $\tau=T_{\mathbb{R}} W \otimes \mathbb{C} \cong T W \oplus \bar{T} W$, and $\bar{\partial}$ is the $\bar{\partial}$-operator. It is easy to see [BB] that there exists a connection $D_{-1}$ for $Q$ on $W-S$ lifting $\delta$ :

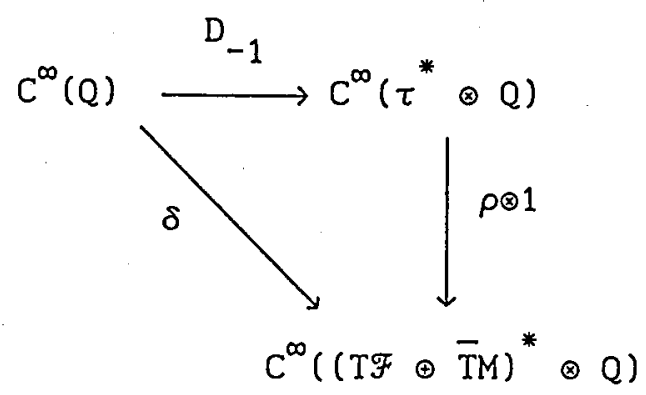

where $\rho$ is the canonical surjection. Any connection on $Q$ lifting the partial connection $\delta$ is called [BB] a basic cannection.

$$
\begin{array}{r}
\text { Now, on } W-S \text { one has a splitting, } \\
T W \cong T \mathscr{T} \oplus Q .
\end{array}
$$

On a neighbourhood $U$ of the boundary $\partial W$, TF has the flat connection $D_{1}^{\prime}$, determined by the vector field $x$. This can be 
extended to a connection $D_{1}$ on $W$, by $[B B ; 4.41]$, so one has a connection $D_{0}^{\prime}=D_{1} \oplus D_{-1}$ for $T W$ on $W-S$.

Let $\left\{z_{\alpha}\right\}$ be the connected components of the singular set $S$. For each component $Z_{\alpha}$ we choose a regular neighbourhood $u_{\alpha}$ of $z_{\alpha}$ and a compact set $\Sigma_{\alpha}$, so that: i) $u_{\alpha}>\Sigma_{\alpha}>$ Int $\Sigma_{\alpha}>z_{\alpha}$, ii) $u_{\alpha} \cap u=\varnothing$ and iii) $u_{\alpha} \cap u_{\beta}=\varnothing$ if $\alpha \neq \beta$. Then by [BB;4.41], there exists a connection $D_{0}$ on $W$ for $T W$, which coincides with $D_{0}^{\prime}$ on $W-\Sigma$, where $\Sigma=U \Sigma_{\alpha}$.

Thus one has connections $D_{0}, D_{-1}$ and $D_{1}$, such that :

i) $D_{-1}$ is basic for $Q$ on $W-S$.

ii) $D_{1}$ is a connection for $L$ on all of $\dot{W}$, and it is flat relative to the vector field $X$ on a neighbourhood $u$ of the boundary $\partial W$.

iii) $D_{0}$ is a connection for TW on $W$, and $D_{0}=D_{1} \oplus D_{-1}$ on $\omega-\Sigma$.

The connections $D_{j}, j=0,1,-1$, determine curvature matrices $K_{0}, K_{1}$ and $K_{-1}$, for $T W, T F$ and $Q$, respectively. If $\sigma_{j}\left(K_{j}\right)$ is the $i^{\text {th }}$-symmetric function in the entries of $K_{j}$, and $\left[\sigma_{i}\left(K_{j}\right)\right]$ is its class in the de Rham cohomology, then the Chern classes of the corresponding bundles are given by,

$$
\begin{aligned}
& c_{i}(T W)=\left(\frac{\sqrt{-1}}{2 \pi}\right)^{i} \cdot\left[\sigma_{i}\left(K_{0}\right)\right], \\
& c_{1}(T F)=\left(\frac{\sqrt{-1}}{2 \pi}\right) \cdot\left[\sigma_{1}\left(K_{1}\right)\right],
\end{aligned}
$$

and on $W-\Sigma$,

$$
c_{i}(Q)=\left(\frac{\sqrt{-1}}{2 \pi}\right)^{i} \cdot\left[\sigma_{i}\left(K_{-1}\right)\right],
$$

see [BB or Mi]. By $i \mathrm{ii}$ ) above, on $W-\Sigma$ one has,

$$
\sigma_{i}\left(K_{-1}\right)=\sum_{j+k=1}\left(-\sigma_{1}\left(K_{1}\right)\right)^{j} \cdot \sigma_{k}\left(K_{0}\right) \text {. }
$$

We observe that the right hand side is actually defined on all of $W$, so we can think of $i t$ as extending $\sigma_{i}\left(K_{-1}\right)$ over $\Sigma$. If we set 


$$
\omega_{i}=\sum_{j+k=1}\left(-\sigma_{1}\left(K_{1}\right)\right)^{j} \cdot \sigma_{k}\left(K_{0}\right)
$$

over $W$, then $\omega_{i}$ defines the Chern class $c_{i}(Q)$ of the sheaf $Q$ on $W$, see [AH].

The class $\phi(Q)$ is represented by the form $\phi\left[\omega_{1}, \ldots, \omega_{n}\right]$, modulo the normalization constant $(\sqrt{-1} / 2 \pi)^{n}$, which coincides with $\phi\left(\sigma_{1}\left(K_{-1}\right), \ldots, \sigma_{n}\left(K_{-1}\right)\right)$ on $W-\Sigma$. By Bott's vanishing theorem, the latter form vanishes identically on $W-\Sigma$. Thus $\phi\left(\omega_{1}, \ldots, \omega_{n}\right)$ defines a relative cohomology class $\tilde{\phi}(Q)$, which is the class in the statement of 2.1 above. Moreover, the restriction of $\phi\left(\omega_{1}, \ldots, \omega_{n}\right)$ to each $u_{\alpha}$ defines a class in $\mathrm{H}^{2 \mathrm{n}}\left(u_{\alpha}, \partial u_{\alpha} ; \mathbb{C}\right)$, and its image by the composition of Lefschetz duality $\mathrm{H}^{2 \mathrm{n}}\left(u_{\alpha}, \partial u_{\alpha} ; \mathbb{C}\right) \rightarrow \mathrm{H}_{0}\left(u_{\alpha} ; \mathbb{C}\right)$ and the inverse of the natural isomorphism $\mathrm{H}_{0}\left(Z_{\alpha} ; \mathbb{C}\right) \rightarrow \mathrm{H}_{0}\left(U_{\alpha} ; \mathbb{C}\right)$, is the class $\operatorname{Res}_{\phi}\left(F ; Z_{\alpha}\right)$ by definition. Thus we have a). To prove $b)$, note that

Since $D_{1}$ is flat on $u, \sigma_{i}\left(K_{0}\right)=\omega_{i}+\omega_{i-1} \cdot \sigma_{1}\left(K_{1}\right)$ vanishes on $u$ and it represents the relative class $\tilde{c}_{1}(F)$. Hence we see that $\phi\left(\sigma_{1}\left(K_{0}\right), \ldots, \sigma_{n}\left(K_{0}\right)\right)$ represents a relative cohomology class $\tilde{\phi}(\mathrm{TW})$ satisfying the identitiy of $b$ ). In particular, if $\phi=c_{n}$, we have

$$
\tilde{c}_{n}(T W)=\tilde{c}_{n}(Q)+c_{n-1}(Q) \cdot \tilde{c}_{1}(F) .
$$

Thus, to complete the proof of 2.1 we only need to show that the above class $\tilde{c}_{n}(T W)$ is the Chern class $\tilde{c}_{n}(T W, X)$ of $\S 1$. For this we recall, from $\$ 1$ above, that the difference $d\left(c_{n}, c_{n}^{\prime}\right)$ between two relative Chern classes $c_{n}, c_{n}^{\prime}$ is given by a contribution of the coboundary map,

$$
\delta^{*}: H^{2 n-1}(\partial W ; \mathbb{Z}) \rightarrow H^{2 n}(W, \partial W ; \mathbb{Z}) .
$$

On a neighbourhood $U$ of $\partial W$. the sheaf $Q$ is actually a vector bundle, and one has a splitting $\left.\left.\left.T W\right|_{u} \cong Q\right|_{U} \oplus T F\right|_{u}$. On $u$, the class $\tilde{c}_{n}(Q)$ is identically 0 , because $Q$ has rank $n-1$. Hence on $u$ one has, 


$$
\tilde{c}_{n}(T W)=\tilde{c}_{1}(F) \cdot c_{n-1}(Q) .
$$

The result follows because $\tilde{c}_{1}(F) c_{n-1}(Q)=\tilde{c}_{n}(T W, X)$ on $u$, since $Q$ is a vector bundle there, and in both cases the characteristic class in question is relative because it is the obstruction for extending the vector field $X$ to the interior of W.

Note that since $\tilde{c}_{n}(T W), \tilde{c}_{1}(F)$, and $c_{n-1}(Q)$ are integral classes, $\tilde{c}_{n}(Q)$ is also integral and the identity in c) holds in $H^{2 n}(W, \partial w ; \mathbb{Z})$.

We now have Theorem I, stated in the introduction:

THEOREM I: Let $X$ be a holomorphic vector field, defined and non-singular on a neighbourhood of $\partial \mathrm{W}$, the boundary of a complex manifold $W$ which is the interior of a compact, smooth manifold with non-empty boundary. Then the total (Poincare-Hopf) index of $X$ in $W$ is :

$$
\operatorname{Ind}(X, W)=\tilde{c}_{1}(F) \cdot c_{n-1}(Q)[W]+\sum_{Z c S} \mu_{*} \operatorname{Res} c_{n}(F, Z)[W] .
$$

where $[W]$ is the fundamental cycle. Moreover, if the singular set $S$ consists of isolated points $\mathrm{P}_{1}, \ldots, \mathrm{P}_{\mathrm{r}_{\mathrm{r}}}$, then

$$
\operatorname{Ind}(X, W)=\tilde{c}_{1}(F) \cdot c_{n-1}(Q)[W]+\sum_{i=1}^{r} \mu_{i},
$$

where $\mu_{i}$ is the local Milnor number of $\mathscr{F}$ at the singular point $P_{i}, i=1, \ldots, r$.

The first statement in this theorem follows from 2.1 together with the fact, noted in $\$ 1$ above, that one has $\tilde{c}_{n}(T W)[W]$ $=$ Ind $(X, W)$. The second claim follows from this and the fact [BB], that at each singular point $P_{i}$, the Baum-Bott residue agrees 
with the corresponding Grothendieck residue, which equals the intersection number $\mu_{i}$. Alternatively, one can prove the second statement via differential topology: Away from the points $P_{1}, \ldots, P_{r}, Q$ is a vector bundle; Therefore, away from these points, the class $c_{n-1}(Q)$ is the first obstruction for constructing a cross section $s$ of $Q,\left(C^{\infty}-\right)$ transversal to the zero section of $Q$. Hence the zero-locus of $\theta$ is a smooth submanifold $\Sigma_{Q}$ of $W-\left\{P_{1}, \ldots, P_{r}\right\}$ of real dimension 2 . This submanifold may extend to the boundary of $W$, so it represents a homology class $\left[\Sigma_{Q}\right] \in \mathrm{H}_{2}\left(W^{*}, \partial W ; \mathbb{Z}\right)$, where $W^{*}$ is $W$ minus small open balls $D_{1}, \ldots, D_{r}$ around the singular points. The natural map from $\mathrm{H}_{2}\left(W^{*}, \partial W ; \mathbb{Z}\right)$ into $\mathrm{H}_{2}(W, \partial W ; \mathbb{Z})$ is an isomorphism, because one has the exact sequence of the triple $\left(W, W^{*}, \partial W\right)$,

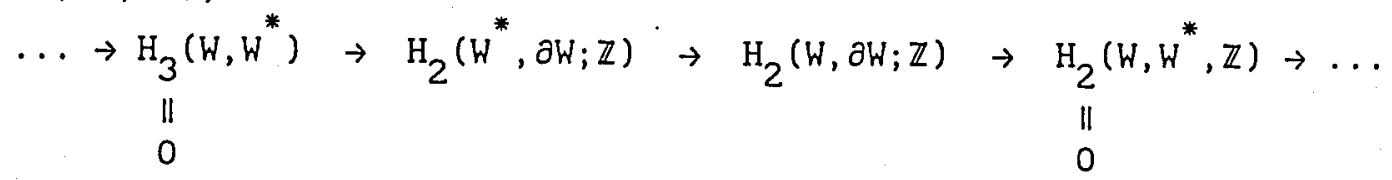

Thus $\Sigma_{Q}$ actually represents a homology class $\left[\Sigma_{Q}\right] \in \mathrm{H}_{2}(W, \partial W ; \mathbb{Z})$, which is isomorphic to $\mathrm{H}^{2 n-2}(W ; \mathbb{Z})$, by Poincaré-Lefschetz duality. Its dual is the Chern class $c_{n-1}(Q)$. Similarly, the class $\tilde{c}_{1}(F)$ is dual to the $(2 n-2)$-submanifold $\Sigma_{\mathscr{F}}$ of $W$, obtained by intersecting the zero-section of $\left.T \mathcal{F}\right|_{W^{*}}$, with a non-zero section $\varphi$ of this bundle, which is transversal to the zero-section and restricts to $X$ on $\partial W$. Since $X$ is non-zero on $\partial W, \Sigma_{F}$ actually represents a class in $\mathrm{H}_{2 n-2}\left(W^{*} ; \mathbb{Z}\right)$, which is isomorphic to $\mathrm{H}_{2 \mathrm{n}-2}(W ; \mathbb{Z}) \cong \mathrm{H}^{2}(W, \partial W ; \mathbb{Z})$. The above intersection number $\tilde{c}_{1}(F) \cdot c_{n-1}(Q)[W]$ is the transverse intersection of $\Sigma_{Q}$ with $\Sigma_{\mathcal{F}}$. We may extend $\varphi$ to the interior of the balls $D_{1}, \ldots, D_{r}$, being tangent to $F$ and singular only at $\mathrm{P}_{1}, \ldots, \mathrm{P}_{r}$, so its local index at each $P_{i}$ is the local Milnor number $\mu_{i}$, by [CLS or GSVl. We may also multiply $s$ by an adequate bump function, to make it zero on $\partial W$ and on the boundary spheres $\partial D_{1}, \ldots, \partial D_{q}$. Thus, adding up the vector fields $s$ and $\varphi$, we obtain a vector field $x$ on $W$ that agrees with $X$ near $\partial W$, it is tangent to $\mathscr{F}$ near the singular points $\mathrm{P}_{1}, \ldots, \mathrm{P}_{\mathrm{r}}$, and its 
remainning singularities are the intersection points $\Sigma_{Q} \cap \Sigma_{\mathscr{F}}$. Hence,

$$
\operatorname{Ind}(X, W)=\operatorname{Ind}(X, W)=\tilde{c}_{1}(F) \cdot c_{n-1}(Q)[W]+\sum_{i=1}^{\Gamma} \mu_{i},
$$

as stated.

We remark that this also provides a new proof of the well known fact, that the Grothendieck residue of $F$ at an isolated singularity $P_{i}$, is the intersection number

$$
\mu_{i}=\operatorname{dim} \frac{o_{n, p}}{\left(F_{1}, \ldots, F_{n}\right)} \text {, }
$$

where $F=\left(F_{1}, \ldots, F_{n}\right)$ is a (local) holomorphic vector field on $W$ at $P$, that spans $F$ on a neighbourhood of $P:$ In this case we take $W$ to be a small neighbourhood $D$ of $P$. Theorem II says,

$\operatorname{Ind}(F ; W)=\mu_{*}\left(\operatorname{Res}_{c_{n}}(F, P)\right)[W]$,
since $\tilde{c}_{1}(F)$ and $c_{n-1}(Q)$ vanish because $D$ is contractible. The left hand side is the local index of $F$ at $P$, and this equals the Milnor number $\mu$. The right hand side equals the corresponding Grothendieck residue, by [BB].

Adding up theorems I and II we arrive to Theorem III, stated in the introduction:

THEOREM III. Let $\mathrm{n}=2 \mathrm{k}$ be even. Let $\mathrm{q}_{\mathrm{n}}$ be the coefficient of $c_{n}$ in $T d_{n}$. Let $(V, P)$ be an ICIS of dimension $\mathrm{n}$, and let $\mathrm{X}$ be a holomorphic vector field on $v$, non-singular away from $P$. If $\tilde{F}$ is the strict transform of the foliation on $V-\{P\}$ determined by $X$, then the index of $X$ on $V$ at $P$ is:

$$
\operatorname{Ind}(X, V)=\sum_{Z \in S} \mu_{*} \operatorname{Res}_{c_{n}}(\tilde{F}, Z)[\tilde{V}]+\tilde{c}_{1}(F) \cdot c_{n-1}(Q)[\tilde{V}]+\frac{1}{q_{n}}\left(\operatorname{Td}^{*}[\tilde{V}]+p_{g}\right) \text {. }
$$

In particular, if the singularities of $\tilde{F}$ are isolated, then the first term on the right hand side is the sum of the local Milnor numbers. 


\section{REFERENCES}

[AH] M.F. Atiyah and F. Hirzebruch. Analytic cycles on complex manifolds. Topology 1 (1962), 25-45.

[BB] P. Baum and R. Bott. Singularities of holomorphic foliations. J. Diff. Geom. 7 (1972), 279-342.

[BG] Ch. Bonatti and X. Gomez-Mont. The index of holomorphic vector fields on singular varieties $I$. To be published in the proceedings of the International Symposium on Holomorphic Dynamical Systems, Rio de Janeiro, 1992.

[BH] A. Borel and F. Hirzebruch. Characteristic classes and homogeneous spaces III. Am. J. Math. 82 (1969), 491-504.

[CLS] C. Camacho, A. Lins and P. Sad. Topological invariants and equidesingularization for holomorphic vector fields. J. of Diff. Geom. 20 (1984), 143-174.

[CCS] C. Camacho, F. Cano and P. Sad. Absolutely isolated singularities of holomorphic vector fields. Inv. Math. 98 (1989), 351-369.

[GSV] X. Gómez-Mont, J.A. Seade and A. Verjovsky. The index of a holomorphic flow with an isolated singularity. Math. Annalen 291 (1991),737-751.

[HH] G. Hector and U. Hirsch. Intoduction to the geometry of foliations. Braunschweig Wiesbaden: Vieweg 1981.

[Hr] F. Hirzebruch. "Topological methods in algebraic geometry". Springer Verlag 1966.

[Hu] D. Husemoller. Fibre bundles. Springer Verlag.

[Ke] M. Kervaire . Relative characteristic classes. Am. J. of Maths. 79 (1957), 517-558.

[La] H.B. Laufer. On $\mu$ for surface singularities. AMS Proc. Symp. Pure Maths. 30 (1977), part I, 45-49.

[LS] B. Li and J. Seade. Framings on algebraic knots. Quart. J. Math. Oxford (2), 38 (1987), 297-306.

[L1] E. Looijenga. "Isolated singular points on complete intersections". London Math. Soc., Lect. Notes 77 (1984). 
[L2] E. Looijenga. Riemann Roch and smoothings of singularities. Topology 25 (1986), 293-302.

[M1] J. Milnor. "Topology from the differentiable viewpoint." Univ. Press of Virginia (1965).

[M2] J. Milnor. "Singular points of complex hypersurfaces". Annals of Math. Studies 61. Princeton Univ. Press (1968).

[M3] J. Milnor. "Characteristic classes". Annals of Math. Studies. Princeton Univ. Press.

[SS] J. Seade and T. Suwa. Residues and singularities of holomorphic foliations on open manifolds. In preparation.

[Se] J.P. Serre. Groupes algébriques et corpes de classes, Hermann, Paris, 1959.

[St] J.H.M. Steenbrink. Mixed Hodge structures associated with isolated singularities. AMS Proc. Symp. Pure Maths. 40 (1983), part 2, 513-536.

José Seade,

Departamento de Matemáticas,

Instituto Tecnológico Autónomo de México,

Rfo Hondo 1, San Angel, México D.F.

and

Instituto de Matemáticas,

Universidad Nacional Autónoma de Méxlco.

Tatsuo Suwa,

Department of Mathematics,

Hokkaldo University,

Sapporo, Japan. 